\title{
MODIFIED SLATER INTEGRALS FOR AN ION IN A SOLID*
}

\author{
C. MORRISON ${ }^{* *}$, D. R. MASON and C. KIKUCHI \\ University of Michigan, Ann Arbor, Michigan, USA
}

Received 18 April 1967

\begin{abstract}
Expressions for the Slater integrals are derived for an ion of the first transition series when placed in a solid. Reasonable agreement is obtained with experimental data for $\mathrm{Co}^{++}$in spinel.
\end{abstract}

Recent experimental evidence [1-3] has indicated that the Slater parameters for ions of the first transition series when introduced into a solid have a value less than for the free ion. It is possible to explain this decrease by introducing the concept of covalency, but we would like to present an alternative approach which can be used to give a quantitative answer. This is not to suggest that covalency is not responsible for many of the observed effects, merely that there are other possible explanations which need to be considered.

Consider an ion in a solid which has a macroscopic dielectric constant $k$. We assume that within a sphere radius $b$ centered on this ion the dielectric constant is unity and that the radius of this sphere is greater than the radius of the ion but smaller than the shortest interatomic bond. Then within this sphere the potential arising from Coulomb interaction of two electrons situated at distances $r_{1}$ and $r_{2}$ from the origin is given by the usual expression:

$$
V(r)=e^{2} /\left|r_{1}-r_{2}\right|
$$

Expanding this expression in spherical harmonics, we have for $r_{1}<r_{2}$

$$
V\left(r_{1} r_{2}\right)=e^{2} \sum_{l m} \frac{4 \pi}{2 l+1} Y_{l m}^{*}\left\langle\hat{\gamma}_{1}\right\rangle Y_{l m}\left\langle\hat{r}_{2}\right\rangle \frac{r_{1}^{l}}{r_{2}^{l+1}}
$$

and the last term on the right hand side becomes $r_{2}^{l} / r_{1}{ }^{l+1}$ for $r_{1}>r_{2}$. This is the expression for the interaction potential for a free ion.

If we now take into account the effect of the surrounding solid for $r>b$ which has a dielectric

* Work supported in part by contract from Harry Diamond Laboratories, Washington, D.C.

** On Fellowship from Harry Diamond Laboratories, Washington, D.C. constant $k$, then another term is required in the expression of the interaction potential. This arises from the imaging effect of the electronic charge through the surface of the sphere due to the abrupt change in dielectric constant. Observing the boundary conditions at $r=b$, the final expression for the potential becomes

$$
\begin{array}{r}
V^{\prime}\left(r_{1} r_{2}\right)=e^{2} \sum_{l m} \frac{4 \pi}{2 l+1} Y_{l m}^{*}\left\langle\hat{r}_{1}\right\rangle Y_{l m}^{*}\left\langle\hat{r}_{2}\right\rangle \\
\times\left[\frac{r_{1}^{l}}{r_{2}^{l+1}}-\frac{r_{1}^{l} r_{2}^{l}(k-1)(l+1)}{D^{2 l+1}(k+l(k+1))}\right]
\end{array}
$$

We assume that the radial dependence of the wave functions is such that there is no contribution to the potential for $r>b$. If we use wavefunctions of the radial form proposed by Slater [4] then for a $3 \mathrm{~d}$ electron this can be written

$$
\varphi=N r^{2} \exp (-\gamma r)
$$

where $N$ is a normalizing constant.

The Slater integrals are generally expressed as [5],

$$
F^{(\mathrm{K})}=e^{2} \int_{0}^{\infty} \int_{0}^{\infty} f\left(r_{i} r_{j}\right) R_{n l}^{2}\left(r_{i}\right) R_{n l}^{2}\left(r_{j}\right) \mathrm{d} r_{i} \mathrm{~d} r_{j}
$$

where $f\left(r_{i} r_{j}\right)$ is the radial dependence of the poten tial.

If we use the two types of radial function from eq. (1), then our expression for $F(K)$ consists of two terms, one attributable to the free ion, the other due to the effect of placing the ion in a solid. Hence

$$
\frac{F_{\text {free }}^{(2)}-F_{\text {crystal }}^{(2)}}{F_{\text {free }}^{(2)}}=\frac{1260}{299}\left(\frac{k-1}{3 k+2}\right)\left[\frac{4}{b \gamma}\right]^{5}
$$




$$
\frac{F_{\text {free }}^{(4)}-F_{\text {crystal }}^{(4)}}{F_{\text {free }}^{(4)}}=\frac{20 \times 10:}{13}\left(\frac{k-1}{5 k+4}\right)\left[\frac{1}{b r}\right]^{9}
$$

The expression for $F^{(0)}$ has not been presented because this term cannot be determined from experimental measurements as it does not affect the relative energy level separations.

In a recent paper [1] the Racah parameters for $\mathrm{Co}^{++}$in the tetrahedral site of $\mathrm{MgAl}_{2} \mathrm{O}_{4}$ have been shown to be $B=830 \mathrm{~cm}^{-1}, C=3570 \mathrm{~cm}^{-1} \mathrm{com}^{-}$ pared with the free ion values of 900 and $3850 \mathrm{~cm}^{-1}$ respectively. The free ion values calculated by the above method are in good agreement with these figures when the appropriate conversion constants are used [6].

Assuming a dielectric constant of 12 , the expressions (3) and (4) give the same decrease in the Slater integrals as observed experimentally if $b$, that is the radius of the imaginary sphere centered on the transition ion, is chosen to be approximately $1.5 \AA$. This value is not unreasonable considering that $\gamma$ of equation (2) is an approximation which can only be avoided by using the Hartree-Fock wavefunctions. For the tetrahedral site in the spinel structure, the nearest neighbor oxygen ions are at a distance of about $2 \AA$.

We have shown using a phenomenological approach that there should always be some reduction in the values of the Slater integrals for an ion when introduced into a solid. This can be explained assuming an ionic model for the solid and that there exists a spheroidal volume centered on the ion with a radius of the order of magnitude of the ionic radius in which the dielectric constant is unity. A similar calculation to the one outlined above can be performed for the rare earth ions; unfortunately comparison with experiment cannot be made due to the lack of optical data for the free ions.

\section{References}

1. M.Drifford and P. Rigny, Comptes Rend. 263B (1966) 180

2. W. Low, Phys.Rev. 109 (1958) 247.

3. W. Low, Phys.Rev, 109 (1958) 256.

4. J.S.Griffith, The theory of transition-metal ions (Cambridge, 1961) p. 104

5. B.R.Judd, Operator techniques in atomic spectroscopy (McGraw-Hill, 1963) p. 80.

6. H. Watanabe, Operator methods in ligand field theory (Prentice-Hall, 1966) p. 109.

\title{
INTERFERENCE EFFECT IN NUCLEAR MAGNETIC RELAXATION
}

\author{
J. S. BLICHARSKI* \\ Physics Department, University of British Columbia, Vancouver 8, Canada
}

Received 19 April 1967

\begin{abstract}
The interference of different intramolecular interactions in nuclear magnetic relaxation process is considered. It is shown a non-exponential time dependence of nuclear magnetization can appear owing to the interference effect.
\end{abstract}

The purpose of this short communication is to show an influence of the interference of intramolecular interactions on nuclear magnetic relaxation process in liquids. As an example I would like to consider the case of molecules containing two identical nuclei of $\operatorname{spin} I=\frac{1}{2}$. In this case the dipole-dipole interaction $G^{\mathrm{d}}$, the anisotropy of electronic screening $G^{\sigma}[1]$ and the spin-rotatio-

* On leave from the Jagellonian University, Cracow, Poland. nal interaction $G^{r}$ can all contribute to the nuclear relaxation process. In the presence of a strong external magnetic field $H_{0}$ the Zeeman interaction is dominant and the above mentioned interactions can be considered as a time depentend perturbation [e.g. 2]

$$
G(t)=G^{\mathrm{d}}(t)+G^{\sigma}(t)+G^{\mathrm{r}}(t),
$$

where

$$
G^{\mathrm{d}, \sigma}=\sum_{m} A_{-m}^{\mathrm{d}, \sigma} Y_{2, m}(\theta(t), \phi(t)),
$$

